\section{$\underset{\substack{\text { hommes } \\ \text { \& migrations }}}{ }$}

\section{Hommes \& migrations}

Revue française de référence sur les dynamiques

migratoires

1293 | 2011

L'immigration dans les musées

\title{
Un musée de l'émigration ouvert à Rome
}

\section{Olivier Desouches}

\section{CpenEdition \\ Journals}

\section{Édition électronique}

URL : http://journals.openedition.org/hommesmigrations/525

DOI : 10.4000/hommesmigrations.525

ISSN : 2262-3353

\section{Éditeur}

Musée national de l'histoire de l'immigration

\section{Édition imprimée}

Date de publication : 1 septembre 2011

Pagination : 125-127

ISSN : 1142-852X

\section{Référence électronique}

Olivier Desouches, «Un musée de l'émigration ouvert à Rome », Hommes \& migrations [En ligne], 1293 | 2011, mis en ligne le 29 mai 2013, consulté le 22 septembre 2020. URL : http://

journals.openedition.org/hommesmigrations/525; DOI : https://doi.org/10.4000/hommesmigrations. 525

Ce document a été généré automatiquement le 22 septembre 2020.

Tous droits réservés 


\title{
Un musée de l'émigration ouvert à Rome
}

\author{
Olivier Desouches
}

1 Le premier des trois parcours de visite proposés par ce musée de l'émigration italienne s'articule selon cinq moments. Le premier remonte aux $\mathrm{XVI}^{\mathrm{e}}$ et $\mathrm{XVII}^{\mathrm{e}}$ siècles préunitaires, quand Lombards et Toscans s'unissent dans l'Allemagne protestante en tant qu'artistes et artisans. La Russie accueille des architectes du Kremlin et des décorateurs des cathédrales de Moscou et de Saint-Pétersbourg, la France de la Renaissance des jardiniers et des marbriers, des tailleurs ou des soyers. L'exode rural permet à des migrants souvent misérables et analphabètes (46,5 \% de la population du Mezzogiorno à la fin du XIX siècle) d'éviter de devenir brigands. La desserte ferroviaire du col du Brenner (1867) comme les tunnels du Fréjus (1871), du Saint-Gothard (1882) et du Sempione (1905) désenclavent la péninsule.

\section{Un parcours chronologique}

2 La fin des gouvernements de la droite historique (1861-1876) inaugure la massification (14 millions) de l'émigration italienne jusqu'en 1915. L'Europe attire encore $64 \%$ des migrants durant la première décennie. Mais, dès 1886, les Amériques prennent le relais : notamment Buenos Aires, où résident déjà 250000 Italiens en 1905, et Sao Paulo, dont presque la moitié des 260000 habitants sont italiens. Gênes se spécialise comme port à destination de l'Amérique latine. Après une première réaction de réprobation, l'État se rallie en 1888 à la liberté d'émigrer et de faire émigrer. En 1901 est institué le Commissariat général à l'émigration, qui aide les candidats au départ à préparer leur voyage. Mussolini le transformera en 1927 en Direction générale des Italiens à l'étranger. Cette deuxième phase est marquée par des naufrages spectaculaires (l'Utopia en 1891, le Bourgogne en 1898, les deux Lusitania en 1901 et 1915, le Sirio en 1906, le Titanic en 1912, l'Ancona en 1915, le Princesse Mafalda en 1927 et l'Arandora Star en 1940) et des vaisseaux cholériques, qui asphyxient certains de leurs passagers ou les laissent mourir de faim. À leur arrivée à New York et à Boston, les survivants sont pieusement 
pris en charge par la société Saint-Raphaël fondée par $\mathrm{M}^{\mathrm{gr}}$ Scalabrini. La colonisation agricole argentine, les cultures du café et du coton au Brésil offrent du travail aux nouveaux venus. En 1908, la Californie accueille déjà cinq banques italiennes, dont la Bank of America and Italy (qui deviendra la Bank of America). Le 6 décembre 1907, 171 Italiens sont victimes d'une catastrophe minière à Monongah, petite ville de Virginie occidentale, et 136 à Marcinelle (Belgique) en 1956. Des Italiens sont massacrés lors de poussées xénophobes comme à Aigues-Mortes en 1893, comme l'a bien raconté dernièrement Gérard Noiriel ${ }^{1}$.

3 Mais l'intégration progresse graduellement grâce à l'école, aux associations aussi vivaces qu'au pays, à la religion ou à la presse (280 périodiques de langue italienne sont édités hors d'Italie au moment de la Première Guerre mondiale).

4 L'entre-deux-guerres reste marqué par les crimes des mafieux Al Capone sous la prohibition, Frank Costello - auquel Martin Scorsese rend hommage en 2006 dans Les Infiltrés - et Lucky Luciano, véritable créateur du trafic d'héroïne. Le Parrain n'est qu'un des 1057 films tournés à Hollywood de 1928 à 2000 consacrés aux Italiens, dont $73 \%$ d'entre eux donnent d'eux une image négative. Ces crimes s'ajoutent aux attentats d'anarchistes italiens hors de leur pays : Caserio assassin à Lyon du président français Sadi Carnot en 1894, Luigi Luccheni de la princesse Sissi à Genève en 1898, les tentatives avortées contre le roi des Belges Léopold II en 1902 et Frankin D. H. Roosevelt en 1933. Réhabilités en 1977, Nicola Sacco et Bartolomeo Vanzetti sont victimes en 1927 de cette confusion entre crime et anarchisme. En raison de mesures restrictives votées en 1924 aux États-Unis, l'Europe redevient la destination privilégiée des émigrants italiens, parfois bien forcés d'y retourner en raison des guerres : en 1931, un tiers (880 000) des étrangers installés en France sont italiens.

Les Trente Glorieuses continuent d'attirer les flux italiens à $68 \%$ vers l'Europe à reconstruire (ou l'agriculture du sud-ouest de la France). $12 \%$ se dirigent vers l'Amérique du Nord, $12 \%$ vers l'Amérique latine et $5 \%$ vers l'Australie, parfois clandestinement quand, en 1962, 87 Italiens se tuent dans "la passe du Diable" près de Vintimille.

Le cinquième temps du premier itinéraire dresse enfin le portrait des Italiens hors d'Italie, masculins à $75 \%$ et travailleurs à $80 \%$, établis aux États-Unis (qui ont accueilli au total 5,8 millions d'émigrants italiens), en France $(4,4)$, en Suisse $(4,3)$, en Argentine et en Allemagne ( 3 chacune) ou au Brésil $(1,5)$. Il y a aujourd'hui dans le monde entre 60 et 70 millions de descendants d'Italiens, dont 24 millions en Argentine, soit la moitié de sa population!

\section{Les apports de cette émigration à l'Italie}

Plus géographique, le deuxième parcours spécifie les régions bénéficiaires des transferts d'argent de ces migrants, au départ souvent affamés : Vénétie (3,2 millions de migrants), Sicile et Campanie (2,9 chacune), Lombardie (2,5), Piémont et Val d'Aoste $(2,3)$, Frioul $(2,2)$ et Calabre (2). Les images de ces diasporas proposées aux visiteurs valident l'hypothèse d'une certaine homogénéité culturelle pour celles originaires $\mathrm{du}$ Mezzogiorno, notamment insulaire. Au contraire, celles plus septentrionales semblent davantage s'adapter aux particularités des pays d'accueil. 
Délibérément interactif (vidéos historiques ou d'archives, chants de migrants et témoignages audio), le voyage s'achève par une dernière étape: l'italianité hors frontières, qu'illustrent des articles de la presse italienne à l'étranger, des maquettes de bateaux à vapeur, des courriers timbrés, des vers de Giovanni Pascoli sur l'errance ou des objets personnels symboliques comme des valises. Voilà qui dissipe les stéréotypes sur la dolce vita ou le design. Les publicités exposées ici vantent les figues de Cosenza vendues en France, l'huile d'olive extra-vierge de Lucques exportée vers 1905, les macaronis siciliens de Giovanni di Cola, la liqueur milanaise Fernet-Branca, goûtée en France dans les années cinquante et soixante, exportée dès 1890 en Amérique du Sud puis en 1902 aux États-Unis, au Mexique, au Canada et à Cuba. Sujet muséal assez novateur, l'émigration permet de réintroduire dans l'histoire transalpine les traditions rurales et une patrimonialisation ethnologique de la mémoire des Italiens. Promu par le ministère des Affaires étrangères en collaboration avec celui des Biens et Activités culturels, ce dispositif muséographique assez classique prévoit de présenter ses collections en région ou à l'étranger. Il complète les registres d'immigrés italiens proposés par le site internet d'Ellis Island qui y ont transité avant leur arrivée à New York, les récits et le documentaire ${ }^{2}$ sur la mémoire d'immigrés italiens et juifs de New York recueillis par Georges Pérec, les photographies d'immigrées italiennes d'Augustus Sherman, les madones de Lewis Hine ou les clichés d'immigrants de Jacob Riis.

Un centre de documentation réunissant en libre accès 500 volumes recueille toute trace écrite du phénomène migratoire. Le visiteur peut enfin visionner un documentaire sur un thème qui, de Rocco et ses frères de Visconti au Christ s'est arrêté à Eboli de Francesco Rosi adapté du roman de Carlo Levi, en passant par Golden Door d'Emmanuele Crialese, Pain et chocolat ou Good morning, Babilonia des frères Taviani, suscite l'intérêt de nombreux cinéastes italiens. Le nôtre aussi, pari gagné.

\section{NOTES}

1. Gérard Noiriel, Le Massacre des Italiens. Aigues-Mortes, 17 août 1893, Paris, Fayard, 2010.

2. Georges Perec, Robert Bobert, Récits d'Ellis Island, histoires d'errance et d'espoir, Paris, Ina, 1979, et P.O.L., 1994 .

\section{RÉSUMÉS}

En 2011, l'Italie célèbre les 150 ans de son unité compliquée et encore contestée au Vittoriano, énorme "machine à écrire" dont l'érection au centre de Rome fut achevée il y a un siècle entre le Capitole, Fori Imperiali et la place de Venise hantée par le souvenir de Mussolini. Honorant le roi 
Victor-Emmanuel II comme le Vittoriale, citadelle de Gabriele d'Annunzio nichée sur le lac de Garde, ce lieu de mémoire ajoute un musée de l'émigration, inauguré en septembre 2009, aux neuf existants déjà régionalement ou localement, et aux six centres d'étude, de documentation ou fondations qui s'y consacrent.

\section{AUTEUR}

\section{OLIVIER DESOUCHES}

Post-doctorant en sociologie, université La Sapienza de Rome, Musée national de l'émigration italienne 